\title{
Ensuring that the diagnosis of tuberculosis accelerates progress towards the Millennium Development Goals
}

\author{
Christy Hanson ${ }^{1}$, Giovanni Sotgiu² and Robert Loddenkemper ${ }^{3}$
}

\begin{abstract}
Affiliations: 'Institute for Global Citizenship, Macalester College, Saint Paul, MN, USA. ${ }^{2}$ Clinical Epidemiology and Medical Statistics Unit, Dept of Biomedical Sciences, University of Sassari, Research, Medical Education and Professional Development Unit, AOU Sassari, Sassari, Italy. ${ }^{3}$ German Central Committee against Tuberculosis, Berlin, Germany.
\end{abstract}

Correspondence: Christy Hanson, Institute for Global Citizenship, Macalester College, 1600 Grand Ave, St Paul, MN 55105, USA. E-mail: chanson5amacalester.edu

0

@ERSpublications

If the goal is zero deaths due to tuberculosis, then no patient can go undiagnosed

http://ow.ly/vdxiM

Global tuberculosis control has been riding a wave of progress over the past decade. Mortality and incidence are now falling in all six World Health Organization (WHO) regions and the tuberculosis-related targets of the Millennium Development Goals now seem more reachable [1,2]. This progress reflects important momentum on many fronts, from more systematic community engagement to increased funding availability. Improvements in tuberculosis case detection have been greatly facilitated by the introduction of new diagnostic technologies and approaches [3-5]. These novel methods are beginning to address clinical, public health and technological challenges, such as diagnosing tuberculosis in people living with HIV, as well as patient barriers to accessing sophisticated technologies and diagnostic results [5-7]. Prior to 2007, no new diagnostic technologies had been endorsed by WHO in decades [8]. However, in the past 5 years, WHO has evaluated and endorsed six diagnostic technologies and methods (table 1) [9]. It has refined its specifications for sputum collection and case identification using smear microscopy, reducing the burden on patients and laboratory technicians. It has proposed diagnostic algorithms to streamline the concurrent availability of various technologies and to improve the sensitivity of screening of people living with HIV. In 2011, WHO went so far as to issue negative recommendations on serodiagnostic tests [10] and interferon- $\gamma$ release assays for high-incidence countries [11], steering national tuberculosis programmes (NTPs) away from diagnostic technologies not yet refined enough to warrant a change in practice [12].

Two articles in a recent issue of the European Respiratory Journal focused on the most recently WHOendorsed diagnostic technology, the Xpert MTB/RIF system (Cepheid, Sunnyvale, CA, USA) [13, 14]. This automated real-time nucleic acid amplification technology was initially recommended in 2010 as a rapid diagnostic test for tuberculosis and rifampicin resistance, and is suitable for use in peripheral as well as higher level laboratories [15, 16]. In its 2013 report, WHO noted that by end of June 2013, 1402 machines and 3.2 million cartridges had been purchased in 88 countries; 94000 multidrug-resistant (MDR) TB cases were diagnosed ( 84000 were culture confirmed and 10000 were identified using the rapid technique) [1].The aforementioned articles published in the European Respiratory Journal summarise the rapid roll-out process [13], and provide one of the most robust cost analyses to date for Xpert MTB/RIF [14]. These timely reviews and economic analyses can inform efforts to retool NTPs as new drugs, diagnostics and vaccines become available. 


\section{TABLE 1 Diagnostic technologies and methods endorsed by the World Health Organization (WHO)}

\section{Date of issue of WHO recommendation}

\section{Diagnostic technology}

or approach assessed

\section{WHO recommendation}

2008

2011

2011

2011

2011

2011

2011
Molecular LPAs

Conventional culture, noncommercial culture and DST

Fluorescent LED microscopy

Same-day diagnosis by microscopy

Xpert MTB/RIF system

\section{Commercial} serodiagnostic tests IGRAs
Only for direct application on smear-positive specimens

Can be used as a stand-alone diagnostic for MDR-TB

Suitable for national or regional laboratories

Both solid and liquid conventional cultures are recommended for national or regional laboratories

Culture is current gold standard and needed for definitive diagnosis of smear-negative TB Culture is required to monitor response to MDR-TB treatment

DST is required for diagnosis of XDR-TB

Specific interim recommendations for non-commercial culture are for CRI methods, MODS and NRA; these are for use only on smear-positive specimens

Should be phased in using a carefully phased implementation plan, eventually replacing conventional microscopy in all settings

Phase-in requires training, validation, quality control, and monitoring and evaluation

Equivalent accuracy to conventional case-finding by microscopy

Recommended change to same-day diagnosis in countries with implemented twospecimen case-finding; countries using three-specimen strategy should consider gradual change with infrastructure improvements

Change to same-day diagnosis must be supported by same-day patient referral to treatment and streamlined lab operations and procedures, including internal and external quality monitoring

Should be used as initial diagnostic test for MDR-TB or TB/HIV suspects; can be used as a stand-alone diagnostic test

Can be considered as follow-on test to microscopy where MDR-TB or HIV is lesser concern, especially in testing smear-negative specimens; this is a conditional recommendation acknowledging major resource implications

Can be used at lower levels (e.g. district/sub-district)

Requires uninterrupted and stable electrical power supply

Should be supported by conventional microscopy, culture, and DST for monitoring of treatment, diagnosis of non-RIF resistance, and surveillance

Very low quality of evidence; these tests should not be used in individuals suspected of active pulmonary or extrapulmonary TB, regardless of HIV status

Insufficient data and low quality of evidence; IGRAs and TST cannot accurately predict the risk of infected individuals developing active TB disease, and should not be used in TB diagnosis

Because of the increased cost and comparable performance of the IGRA compared to the TST, replacement of TST with IGRA in resource-constrained settings is not recommended

All tuberculosis (TB) diagnostic capacity should be linked to drug access and programmatic capacity to ensure treatment of patients under appropriate international standards of care. Country-specific algorithms are dependent on several factors, including local epidemiology, local treatment policies, existing country laboratory capacity, specimen referral and transport mechanisms, and availability of human and financial resources. LPA: line probe assay; MDR: multidrug-resistant; DST: drug susceptibility testing; XDR: extensively drug-resistant; CRI: colourimetric redox indicator; MODS: microscopic observation drug susceptibility; NRA: nitrate reductase assay; LED: light-emitting diode; RIF: rifampicin; IGRA: interferon- $\gamma$ release assay; TST: tuberculin skin test.

Looking to the future, it is worth reflecting on what the current articles' findings and discussions teach us about the introduction of a new tool. First, we turn our attention to the types of evidence that guide decision making. Conceptually, we hope that diagnostic technologies will facilitate improved case detection as well as rapid detection of rifampicin resistance. To be effective, tuberculosis diagnostic technologies must be affordable for disease-endemic, high- and low-income countries, accessible to patients, acceptable to both patients and laboratory technicians, appropriate for use in the local infrastructure, and fitting to the epidemiological scenario [12]. It is not enough for a new TB diagnostic technology to be highly sensitive and specific under controlled test conditions. Successful new diagnostic technologies must demonstrate a significant advantage as a complement to, or replacement of, existing diagnostic tools; only tools that enhance case detection (including the detection of drug resistances) warrant the major investments of human and financial resources required to adopt and introduce them. Advantages may come through myriad channels, including cost savings, more rapid results, reduced infectiousness of the case, and/or improved sensitivity and/or specificity [17]. To assess new technologies and approaches from this broad 
perspective, the complexity of evidence required cannot be underestimated. Yet, the current articles reflect that it is possible to comply with systematic attention to this need for evidence.

The papers reflect important developments in the evaluation of new diagnostics that have moved the field forward. As highlighted in the article by WeYER et al. [13], in 2008, WHO adopted the GRADE (Grades of Recommendations Assessment, Development and Evaluation) process for evaluating scientific evidence on new TB findings [18]. While laborious, this process prompts a multifaceted consideration of evidence and encourages consideration of field/practice appropriateness. In 2009, the New Diagnostics Working Group of the Stop TB Partnership, in collaboration with WHO, published Pathways to Better Diagnostics for Tuberculosis: a Blueprint for the Development of TB Diagnostics [19]. This publication suggested that diagnostic development needs to include consideration and assessment of a tools' effectiveness throughout the process of implementation and scale-up, not just the evaluation of purely technical parameters. These two developments began to merge the formerly disparate worlds of research and development, and programme implementation, in a highly constructive manner. The process for reviewing Xpert MTB/RIF benefitted from the availability of some, albeit limited, evidence from field-level implementation of the technology. The resulting WHO endorsement was highly nuanced, offering countries more than a "thumbs up" on the technology, by elaborating the variable strength of the recommendations for use of the technology in different epidemiological and field settings. NTPs could begin to see where the technology might enhance their case detection efforts.

Yet, questions about implementation were prevalent following the WHO endorsement. There were initial and persistent concerns about the appropriateness of Xpert MTB/RIF given its costs, technological requirements and specifications [20]. As described by the current articles, much of the early roll-out of Xpert MTB/RIF incorporated operational research that addressed these initial concerns. This allowed growth of the evidence base from field use. Clearly, the affordability, accessibility and appropriateness of diagnostic tools must be considered as part of the WHO review and endorsement process. However, we have learned that there can be a balanced approach to enable immediate roll-out when and where the intervention is of clear benefit, while systematic evaluation of implementation is sought to inform improvements of the technology and guide further roll-out. In the case of the Xpert MTB/RIF, PANTOJA et al. [14] have documented that the costs and affordability of using Xpert MTB/RIF can offer efficiency gains for some programme settings. This evidence was simply not available when an initial decision had to be made about recommending the tool, but its availability will surely enhance NTP decision making.

One of the most decisive messages that we take from these reviews is that countries are eager and able to rapidly adopt and introduce new technologies that hold promise for improving their programmatic outcomes. The scale-up of the standard DOTS (directly observed therapy, short course) approach [21] and the introduction of fixed-dose combination drugs $[22,23]$ are examples of programmatic retooling that took many years to roll out. With 88 countries having adopted and introduced machines to detect rifampicin resistance within $\sim 3$ years of its endorsement, it is fair to assume that future technological improvements are eagerly anticipated and will be in high demand [24]. The time from policy to practice has dramatically decreased, as roadmaps for the adoption, introduction and uptake of technologies have proven successful $[19,25]$. The commitment of disease-endemic countries to improving access and care for patients must be met with dynamic processes for guideline development, technical assistance, planning for adequate supplies and flexible funding. Where the worlds of research and development, and programme implementation have now come together around the generation of evidence, the worlds of laboratory networks, NTPs and health systems experts must also now come together to support optimised use of new tools.

WEYER et al. [13] aptly note that "One size no longer fits all, and expert laboratory input is needed to define the most cost-effective and efficient algorithms in individual countries" [13]. The need for country-specific diagnostic algorithms may become one of the greatest challenges. Six new diagnostic technologies receiving WHO endorsements in 5 years have left NTPs scrambling to adapt and improve continuously [9]. Many of the new diagnostic technologies under development will not necessarily replace old technologies. They will complement existing tools and/or best suit particular health system contexts or epidemiological realities. The tuberculosis community lived so long with such limited choices that we are struggling with the novel menu of options. PANTOJA et al. [14] highlighted where Xpert MTB/RIF can be cost efficient and even cost saving in particular situations. This type of evidence will support NTP-level decision making for the adoption of the tool and perhaps planning about its efficient placement. However, it will take collaboration between laboratory experts and tuberculosis programmatic/health systems experts in each country to define the algorithms that will enable implementation that optimises the use of the tool for improving case detection, including drug resistance testing.

The need for technical assistance to define context-specific diagnostic algorithms that incorporate the use of Xpert MTB/RIF was discussed by WeYER et al. [13]. The tuberculosis community has not previously had to 
enable this magnitude of adaptation of global guidance. Appropriate technical assistance will need to come from a place of context-specific experience and expertise. Often, the best experience may come from a neighbouring country, particularly where infrastructure and epidemiology are similar. Perhaps the next shift of the global architecture will nurture more systematic south-to-south exchange of technical assistance and shared guidance to support the roll-out of new tools. Therefore, it is to be hoped that new political activities fostering collectivism and cooperation among high tuberculosis incidence countries are implemented and scaled up.

Despite the optimism raised by these articles, we must remember that WHO estimated that 1.3 million people died due to tuberculosis in 2012 [1]. This is unacceptable [26]. If the final goal is to reach zero deaths due to tuberculosis, it should be unanimously accepted that no patient can go undiagnosed. We are still waiting for the shining star, a point-of-care diagnostic that does not discriminate based on HIV status, race, age or sex. In the meantime, we applaud the dynamic and evidence-based approach taken by countries and the global community to adopt what works, continuing to refine diagnostic algorithms for patients seeking care.

\section{References}

World Health Organization. Global tuberculosis report 2013. WHO/HTM/TB/2013.11. Geneva, WHO, 2013.

United Nations. Millennium Development Goals Report. New York, United Nations, 2011.

Keeler E, Perkins MD, Small P, et al. Reducing the global burden of tuberculosis: the contribution of improved diagnostics. Nature 2006; 444: Suppl. 1, 49-57.

4 Raviglione M, Marais B, Floyd K, et al. Scaling up interventions to achieve global tuberculosis control: progress and new developments. Lancet 2012; 379: 1902-1913.

5 Pai M, Palamountain KM. New tuberculosis technologies: challenges for retooling and scale-up. Int J Tuberc Lung Dis 2012; 16: 1281-1290.

6 Drobniewski F, Nikolayevskyy V, Balabanova Y, et al. Diagnosis of tuberculosis and drug resistance: what can new tools bring us? Int J Tuberc Lung Dis 2012; 16: 860-870.

7 Peter JG, Theron G, van Zyl-Smit R, et al. Diagnostic accuracy of a urine lipoarabinomannan strip-test for TB detection in HIV-infected hospitalised patients. Eur Respir J 2012; 40: 1211-1220.

8 Keshavjee S, Farmer PE. Tuberculosis, drug resistance, and the history of modern medicine. N Engl J Med 2012; 367: 931-936.

9 World Health Organization. Policy Framework for Implementing TB Diagnostics. Geneva, WHO, 2011.

10 World Health Organization. Commercial serodiagnostic tests for diagnosis of tuberculosis: policy statement. WHO/HTM/TB/2011.5. Geneva, WHO, 2011.

11 World Health Organization. Use of tuberculosis interferon-gamma release assays (IGRAs) in low- and middleincome countries: policy statement. WHO/HTM/TB/2011.18. Geneva, WHO, 2011.

12 Trébucq A, Enarson DA, Chiang CY, et al. Xpert® MTB/RIF for national tuberculosis programmes in low-income countries: when, where and how? Int J Tuberc Lung Dis 2011; 15: 1567-1572.

13 Weyer K, Mirzayev F, Migliori GB, et al. Rapid molecular TB diagnosis: evidence, policy making and global implementation of Xpert MTB/RIF. Eur Respir J 2013; 42: 252-271.

14 Pantoja A, Fitzpatrick C, Vassall A, et al. Xpert MTB/RIF for diagnosis of tuberculosis and drug-resistant tuberculosis: a cost and affordability analysis. Eur Respir J 2013; 42: 708-720.

15 World Health Organization. Policy Statement: Automated Real-time Nucleic Acid Amplification Technology for Rapid and Simultaneous Detection of Tuberculosis and Rifampicin Resistance: Xpert MTB/RIF System. WHO/ HTM/TB/2011.4. Geneva, WHO, 2011.

16 World Health Organization. Rapid Implementation of the Xpert MTB/RIF Diagnostic Test. Technical and Operational "How-to" Practical Considerations. WHO/HTM/TB/2011.2. Geneva, WHO, 2011.

17 Floyd K, Hutubessy R, Kliiman K, et al. Cost and cost-effectiveness of multidrug-resistant tuberculosis treatment in Estonia and Russia. Eur Respir J 2012; 40: 133-142.

18 Guyatt GH, Oxman AD, Vist GE, et al. GRADE: an emerging concensus on rating quality of evidence and strength of recommendations. BMJ 2008; 336: 924-926.

19 World Health Organization, Stop TB Partnership. Pathways to better diagnostics for tuberculosis: a blueprint for the development of TB diagnostics. Geneva, WHO, 2009.

20 Meyer-Rath G, Schnippel K, Long L, et al. The impact and cost of scaling up GeneXpert MTB/RIF in South Africa. PLoS One 2012; 7: e36966.

21 Espinal MA, Dye C, Raviglione M, et al. Rational "DOTS plus” for the control of MDR-TB. Int J Tuberc Lung Dis 1999; 3: 561-563.

22 Gautam CS, Saha L. Fixed dose drug combinations (FDCs): rational or irrational: a view point. Br J Clin Pharmacol 2008; 65: 795-796.

23 Albanna AS, Smith BM, Cowan D, et al. Fixed-dose combination antituberculosis therapy: a systematic review and meta-analysis. Eur Respir J 2013; 42: 721-732.

24 Barnard M, Warren R, Van Pittius NG, et al. GenoType MTBDRsl Line Probe Assay shortens time to diagnosis of extensively drug-resistant tuberculosis in a high-throughput diagnostic laboratory. Am J Respir Crit Care Med 2012; 186: 1298-1305.

25 Pai M, Minion J, Steingart K, et al. New and improved tuberculosis diagnostics: evidence, policy, practice, and impact. Curr Opin Pulm Med 2010; 16: 271-284.

26 Caminero JA, Matteelli A, Loddenkemper R. Tuberculosis: are we making it incurable? Eur Respir J 2013; 42: 5-8. 